\title{
Steady-State Pharmacokinetics of MNK-795, an Extended-Release Oxycodone and Acetaminophen Combination Analgesic: Results from 2 Active Comparator Studies
}

\author{
Krishna Devarakonda*, Terri Morton, Michael Giuliani, Kenneth Kostenbader and Thomas Barrett
}

Mallinckrodt Inc., Hazelwood, MO, USA

\begin{abstract}
Two single-center, open-label, randomized, phase 1, multiple-dose studies ( $\mathrm{N}=48$ each) evaluated the steadystate pharmacokinetics and tolerability of MNK-795, a bilayer product with both immediate-release (IR) and extendedrelease (ER) components of oxycodone (OC) and acetaminophen (APAP; OC/APAP ER). Study 1 compared 1- and 2-tablet doses of OC/APAP ER $(7.5 \mathrm{mg} / 325 \mathrm{mg} ; 15 \mathrm{mg} / 650 \mathrm{mg})$ administered every 12 hours and 1 tablet of IR OC/ APAP $(7.5 \mathrm{mg} / 325 \mathrm{mg})$ administered every 6 hours over 4.5 days. Study 2 compared OC/APAP ER administered as 2 tablets $(15 \mathrm{mg} / 650 \mathrm{mg}$ ) every 12 hours with IR oxycodone $(15 \mathrm{mg})$, IR tramadol/APAP $(37.5 \mathrm{mg} / 325 \mathrm{mg})$, and IR OC/APAP $(7.5 \mathrm{mg} / 325 \mathrm{mg})$, each administered as 1 tablet every 6 hours over 4.5 days. In both studies, steady-state, dose-normalized, $\mathrm{AUC}_{0-12 \mathrm{~h}}, \mathrm{C}_{\text {avg }}$, and $\mathrm{C}_{\min }$ for oxycodone were similar between OC/APAP ER and each comparator; however, the degree of fluctuation and swing in oxycodone concentrations were greater with the more frequently dosed IR formulations. Acetaminophen concentrations reached similar peak levels to the IR products, but with OC/ APAP ER acetaminophen concentrations tapered to levels that were below those observed with the IR comparators by 7 hours after the last dose. The most commonly observed adverse events included nausea, vomiting, dizziness, and pruritus.
\end{abstract}

Keywords: Analgesic: Pain; Opioid; Extended-release; Oxycodone; Acetaminophen; Pharmacokinetics

Abbreviations: ANOVA: Analysis of Variance; AE: Adverse Event; APAP: Acetaminophen; AUC: Area under the Plasma Concentration-Time Curve; $\mathrm{AUC}_{0-12 \mathrm{~h}}$ : AUC from time 0 to 12 hours; $\mathrm{AUC}_{0-12 s \mathrm{~s}}:$ Steady-State AUC from Time 0 to 12 hours; $\mathrm{C}_{\text {avgss }}$ : SteadyState Average Observed Plasma Concentration During the Dosing Interval; $\mathrm{C}_{\max }$ : Maximum plasma concentration; $\mathrm{C}_{\operatorname{maxss}}$ : Steady-State $\mathrm{C}_{\max } ; \mathrm{C}_{\min }$ : Minimum Plasma Concentration; $\mathrm{C}_{\text {minss }}$ : Steady-State $\mathrm{C}_{\min }$; CI: Confidence Interval; DFL: Degree of Fluctuation; ECG: Electrocardiogram; ER: Extended-Release; IR=Immediate-Release; $\mathrm{K}_{\mathrm{el}}$ : Apparent First-Order Terminal Elimination Rate Constant; LCMS/MS: Liquid Chromatography-Tandem Mass Spectrometry; LS: Least Squares; OC=Oxycodone; PK: Pharmacokinetics; SAE=Serious Adverse Event; SD: Standard Deviation; Swing: Swing of Plasma Concentrations; $\mathrm{t}_{1 / 2}$ : Elimination Half-Life; $\mathrm{t}_{\mathrm{lag}}:$ Lag time; $\mathrm{T}_{\max }$ : Time to Peak Plasma Concentrations; $\mathrm{T}_{\operatorname{maxs}}=$ Steady-State $\mathrm{T}_{\max }$

\section{Introduction}

Acute pain is common, affecting between 25 and 97 million Americans each year [1-5]. Although several analgesic options exist, acute pain is often undermanaged, and many patients continue to experience pain [5-7]. Opioids are commonly used to manage moderate to severe acute pain $[6,8]$. Oxycodone is a semisynthetic opioid used primarily for its analgesic effects [9], and has demonstrated high oral bioavailability and linear pharmacokinetics (PK) [10]. Immediate-release (IR) formulations of oxycodone have relatively short duration of action, requiring administration every 4 to 6 hours to maintain analgesia, whereas extended-release (ER) formulations are designed to maintain plasma concentrations for longer periods with less frequent dosing [10-12]. Opioids are often used in combination with acetaminophen, a nonopioid, nonsalicylate analgesic/antipyretic that is postulated to exert analgesic effects predominantly via central mechanisms $[13,14]$.
The primary goal of combination therapy is to provide adequate analgesia while limiting side effects of both agents. Combining agents with complementary mechanisms of action, particularly those which are proposed to have additive analgesic effects, allow for lower doses of the individual agents, and thus, may result in a lower risk for concentration-dependent adverse events (AEs) [15-19]. Combination oxycodone/acetaminophen (OC/APAP) therapy with IR products has demonstrated clinical efficac across a variety of conditions associated with moderate to severe pain $[14,15,20-28]$.

MNK-795 (OC/APAP ER) is a combination oxycodone and acetaminophen bilayer analgesic with both IR and ER components designed for 12-hour dosing. OC/APAP ER tablets employ a dual-layer biphasic delivery mechanism that, when administered as a single dose (ie, 2 tablets), is designed to deliver $3.75 \mathrm{mg}$ OC/325 mg APAP through the IR component and $11.25 \mathrm{mg}$ OC/325 mg APAP through the ER component, which releases medication at a steady rate in the upper gastrointestinal tract.

Immediate-release IR-OC/APAP (Percocet) was used initially as a comparator in Study 1. Since Percocet was not approved by the FDA under a New Drug Application (NDA), but rather an Abbreviated New Drug Application (ANDA), the pharmacokinetics of OC and APAP

*Corresponding author: Krishna Devarakonda, Mallinckrodt Inc., 675 James McDonnell Blvd 302-3-W, Hazelwood, MO 63042, USA, Tel: (314) 654-3364; Fax: (314) 654-9364; E-mail: krishna.devarakonda@mallinckrodt.com

Received January 09, 2014; Accepted February 24, 2014; Published March 04 2014

Citation: Devarakonda K, Morton T, Giuliani M, Kostenbader K, Barrett T (2014) Steady-State Pharmacokinetics of MNK-795, an Extended-Release Oxycodone and Acetaminophen Combination Analgesic: Results from 2 Active Comparator Studies. J Bioequiv Availab 6: 053-060. doi:10.4172/jbb.1000180

Copyright: ( 2014 Devarakonda K, et al. This is an open-access article distributed under the terms of the Creative Commons Attribution License, which permits unrestricted use, distribution, and reproduction in any medium, provided the original author and source are credited. 
Citation: Devarakonda K, Morton T, Giuliani M, Kostenbader K, Barrett T (2014) Steady-State Pharmacokinetics of MNK-795, an Extended-Release Oxycodone and Acetaminophen Combination Analgesic: Results from 2 Active Comparator Studies. J Bioequiv Availab 6: 053-060. doi:10.4172/jbb. 1000180

from MNK-795 (7.5 mg OC/325 mg APAP) were also compared to NDA approved immediate-release Roxicodone and Ultracet. In addition, given that the PK comparison between MNK-795 and the IR comparator was still highly relevant for prescribing physicians, Percocet $7.5 \mathrm{mg}$ OC/325 mg APAP was included in this study.

We report results of 2 studies conducted in healthy volunteers to evaluate the steady-state PK of OC/APAP ER. The first study compared 1 - and 2-tablet dosages of OC/APAP ER administered every 12 hours over 4.5 days with 1 tablet dosages of IR OC/APAP administered every 6 hours over 4.5 days. The second study compared OC/APAP ER administered as 2 tablets every 12 hours with IR OC, IR tramadol/ APAP, and IR OC/APAP, each administered as 1 tablet every 6 hours.

\section{Materials and Methods}

\section{Participants}

Inclusion and exclusion criteria were similar in both studies. Healthy men or nonlactating, nonpregnant women, 18 to 55 years of age, with body mass index between 19 and $\leq 30 \mathrm{~kg} / \mathrm{m}^{2}$ and minimum weight of $130 \mathrm{lb}$ were eligible. Exclusion criteria included smoking or use of nicotine-containing products in the previous 6 months; history of drug or alcohol use or positive urine test for drugs of abuse; use of prescription or over-the-counter medications within 14 days of study check-in; history of medication allergy, hypersensitivity, or intolerance of opioid products (including tramadol in study 2) or acetaminophen; history of any condition that may interfere with absorption, distribution, metabolism, or excretion of study medication; or previous gastric bypass or gastric band surgery. Participants with histories of abdominal and/or pelvic surgery within 1 year of study initiation; previous cardiothoracic surgery; histories of psychiatric symptoms or disorders requiring hospitalization, psychotherapy, and/or medication within 3 years of study initiation; acute or chronic gastrointestinal disease; histories of seizures or diagnosis of epilepsy or other seizure disorder or histories of conditions that might be specifically contraindicated or require caution while using oxycodone, acetaminophen, and/or tramadol were also excluded.

\section{Ethical conduct}

These studies were conducted in accordance with Good Clinical Practice guidelines, and the protocol was approved by the investigator's Institutional Review Board (IntegReview, Austin, TX, USA). Written informed consent was obtained from participants before enrollment.

\section{Study design and treatments}

Both studies were single-center, open-label, randomized, phase 1, multiple-dose trials. Study 1 was a 3-period, 6-sequence, crossover study. Participants received each of the following treatments for 4.5 days under fasted conditions: (1) OC/APAP ER ( 1 tablet; $7.5 \mathrm{mg} / 325$ $\mathrm{mg}$ ) taken every 12 hours ( 9 tablets total); (2) OC/APAP ER (2 tablets; total, $15 \mathrm{mg} / 650 \mathrm{mg}$ ) taken every 12 hours (18 tablets total); and (3) IR OC/APAP ( 1 tablet; $7.5 \mathrm{mg} / 325 \mathrm{mg}$ ) taken every 6 hours (18 tablets total). The study included a screening visit and 3 confinement periods of approximately 7 days each, with a minimum of 14 days between the start of each period, and a telephone follow-up of at least 7 days after study completion. Plasma samples for PK analysis were collected at intervals up to 144 hours after the first dose of each confinement period. AEs were monitored throughout each confinement period and during the 7-day follow-up.

Study 2 was a 4 -period, 4 -sequence study in which participants received each of the following treatments under fasted conditions for 4.5 days: (1) OC/APAP ER (2 tablets; total, $15 \mathrm{mg} / 650 \mathrm{mg}$ ) taken every 12 hours (18 tablets total); (2) IR oxycodone (1 tablet; $15 \mathrm{mg}$ ) taken every 6 hours (18 tablets total); (3) IR tramadol/APAP ( 1 tablet; $37.5 \mathrm{mg} / 325$ $\mathrm{mg}$ ) taken every 6 hours (18 tablets total); and (4) IR OC/APAP (1 tablet; $7.5 \mathrm{mg} / 325 \mathrm{mg}$ ) taken every 6 hours (18 tablets total). The study included a screening visit and 4 confinement periods of approximately 7 days each, and 3 intervals of at least 13 days between the start of each period. Plasma samples for PK analysis were collected at intervals up to 132 hours after the first dose of each confinement period. AEs were monitored from the time the informed consent form was signed through either the end of the study or upon early termination. Ongoing AEs were followed by the investigator until the events subsided, values returned to a normal range, follow-up was determined to be no longer necessary, or the participant was referred to his or her usual physician for follow-up.

\section{Plasma sampling and assessments}

Participants fasted overnight for a minimum of 10 hours before the first dose. A meal was provided approximately 4 hours postdose. In study 1, blood samples for PK analysis of oxycodone and acetaminophen were collected by venipuncture on day 1: predose, $15,30,45$ minutes, and 1, 2, 3, 4, 6, 7, 8, 10, and 12 hours postdose, with additional samples collected at 15,30 , and 45 minutes after the 6-hour IR OC/APAP dose; days 2 to 4 : before the morning dose (24, 48 , and $72 \mathrm{~h}$ ); and day 5 to 7 : predose (before hour 96 ), then 15,30 , and 45 minutes after the dose at hour 96, and at 97, 98, 99, 100, 102, 103, $104,106,108,112,120$ (day 6), 132, and 144 hours (day 7). Additional samples were collected 15,30 , and 45 minutes after the dose at 102 hours for IR OC/APAP. In study 2, blood samples for PK analysis of oxycodone and acetaminophen were collected by venipuncture before dosing and at $0.5,1,2,3,4,6,6.5$ (IR formulations only), 8, and 12 hours after dosing on day 1 ; before the morning dose on days 2 through 4 (24, 48, and $72 \mathrm{~h}$ ); before dosing on day 5 (96) and at $96.5,97,98$, 99, 100, 102, 102.5 (IR formulations only), 104, 108, $120 \mathrm{~h}$ (day 6), and 132 hours. In both studies, blood samples were collected into prechilled vacuum collection tubes containing EDTA and placed into a cryoblock immediately after collection. Samples were centrifuged at approximately $4^{\circ} \mathrm{C}$, and the plasma fraction was divided into 2 labeled polypropylene tubes and frozen until analysis using a validated liquid chromatography-tandem mass spectrometry (LC-MS/MS) assay at a bioanalytical laboratory (PPD, Inc., Middleton, Wisconsin, USA). The LC-MS/MS method for oxycodone was validated over a calibration range of 0.100 to $100 \mathrm{ng} / \mathrm{mL}$, and the method for acetaminophen was validated over a calibration range of 100 to $50,000 \mathrm{ng} / \mathrm{mL}$. Internal standards for the quantitative LC-MS/MS method were OC- $\mathrm{d}_{6}$ and APAP- $\mathrm{d}_{4}$. Human plasma containing oxycodone, acetaminophen, and the internal standards was extracted, reconstituted, and injected onto the LC-MS/MS. Each calibration curve was calculated using a linearweighted least squares regression algorithm, and calibration curves were plotted as the peak area of the analyte to the internal standard versus concentration.

Oxycodone and acetaminophen pharmacokinetics were examined using the following parameters: day 1 - area under the plasma concentration-time curve (AUC) from time 0 to 12 hours $\left(\mathrm{AUC}_{0-12 \mathrm{~h}}\right)$, maximum observed plasma concentration $\left(\mathrm{C}_{\max }\right)$, minimum plasma concentration obtained at 12 hours after dosing $\left(\mathrm{C}_{\min }\right)$, time to $\mathrm{C}_{\text {max }}$ $\left(\mathrm{T}_{\mathrm{max}}\right.$ ), lag time (ie, time between administration and the first measurable concentration; $\mathrm{t}_{\mathrm{lag}}$ ); day 5 - steady-state AUC from time 0 to 12 hours 
Citation: Devarakonda K, Morton T, Giuliani M, Kostenbader K, Barrett T (2014) Steady-State Pharmacokinetics of MNK-795, an Extended-Release Oxycodone and Acetaminophen Combination Analgesic: Results from 2 Active Comparator Studies. J Bioequiv Availab 6: 053-060. doi:10.4172/jbb.1000180

$\left(\right.$ AUC $\left._{0-12 h s s}\right)$, steady-state $C_{\text {max }}\left(C_{\text {maxss }}\right)$, steady-state $C_{\text {min }}\left(C_{\text {minss }}\right)$, steadystate average observed plasma concentration during the dosing interval $\left(\mathrm{C}_{\text {avgs }}\right)$, degree of fluctuation in plasma concentrations (DFL), swing of plasma concentrations (swing), steady-state $\mathrm{T}_{\max }\left(\mathrm{T}_{\text {maxss }}\right)$, apparent first order terminal elimination rate constant $\left(\mathrm{K}_{\mathrm{el}}\right)$, and apparent plasma terminal elimination half-life $\left(\mathrm{t}_{1 / 2}\right)$. DFL and swing are parameters used to assess delivery properties of formulations at steady state. DFL was calculated as $\left(\mathrm{C}_{\text {maxss }}-\mathrm{C}_{\text {minss }} / \mathrm{C}_{\text {avgss }}\right) \cdot 100 \%$, and swing was calculated as $\left(\mathrm{C}_{\text {maxss }}-\mathrm{C}_{\text {minss }}\right) / \mathrm{C}_{\text {minss }}^{\text {maxss }}[29]$.

Safety and tolerability were assessed by monitoring AEs and conducting clinical laboratory tests (eg, hematology, serum chemistry, and urinalysis), vital sign and pulse oximetry measurements, electrocardiograms (ECGs), physical examinations, and impaired judgment evaluations.

\section{Statistics}

Data from all-dosed subjects were used for the safety analyses. The completer population was defined as subjects who finished all study periods and was used for the steady-state PK analysis of OC/APAP ER. Descriptive statistics and frequency counts were used for subject disposition, demographics, and baseline characteristics. Summary statistics were compiled for treatment-emergent AEs. Actual values and changes from baseline were assessed by treatment laboratory measures, vital signs, pulse oximetry, and ECGs.

Individual plasma concentration versus actual time data were used to estimate the PK parameters for oxycodone and acetaminophen by standard noncompartmental methods using Phoenix ${ }^{\circledR}$ WinNonlin ${ }^{\circledR}$ (Pharsight Corporation, St. Louis, Missouri, USA) Version 6.1 for each individual in each treatment. PK parameters were summarized by treatment using descriptive statistics, number of subjects, mean, standard deviation (SD), median, minimum, and maximum. Statistical comparisons between conditions at steady-state (day 5) were made for dose-normalized (plasma concentration $\div$ dose administered) PK parameters of $\mathrm{C}_{\text {maxss }}, \mathrm{C}_{\text {minss }}, \mathrm{C}_{\text {avgss }}$, and $\mathrm{AUC}_{0-12 \mathrm{ss}}$, and natural $\log$ transformed PK parameters $A U C_{0-12 h s s}, C_{\text {maxss }}, C_{\text {avgss }}, C_{\text {minss }}$ of DFL and swing, using analysis of variance (ANOVA) with PK parameters defined as dependent variables with sequence, treatment, and period as fixed effects, and subjects nested within sequences as random effects. Comparisons were based on geometric least squares (LS) means, percentage ratio of geometric LS means, and corresponding $90 \%$ confidence intervals (CI). A $90 \%$ CI of geometric LS means ratios fully contained within $80 \%$ to $125 \%$ concluded that there was no difference between treatments.

Non-transformed PK parameters $\left(\mathrm{K}_{\mathrm{el}}\right.$ and $\left.\mathrm{t}_{1 / 2}\right)$ were compared with ANOVA using these PK parameters as the dependent variable, sequence, treatment, and period as fixed effects, and subjects nested within sequences as random effects. LS means, difference of the LS means, $90 \% \mathrm{CI}$ of the difference, and $P$ values for testing fixed effects were calculated. Wilcoxon signed rank tests were used to determine statistical significance of median difference for $\mathrm{T}_{\max }$ at steady-state between treatments at a significance level of $P \leq 0.05$.

In addition, an analysis of the time that steady-state concentrations were reached was performed using ANOVA with the natural logtransformed $\mathrm{PK}$ parameter $\mathrm{C}_{\min }$ defined as the dependent variable, day and sequence as fixed effects, and subjects nested within sequence as random effects. Helmert transformation compared geometric mean concentration of the corresponding study day to the geometric mean concentration pooled over all remaining days within treatment. Comparisons continued until the day the comparison was not statistically significant (ie, $\alpha=0.05$ ). The earliest study day that included a nonsignificant contrast was considered as the study day on which steady-state concentrations were attained. Geometric LS means, percentage ratio of geometric LS means, and corresponding $90 \% \mathrm{CI}$ of the ratio were calculated. All analyses were performed using SAS software (SAS Institute, Inc., Cary, North Carolina, USA), version 9.1

\section{Results}

\section{Participants}

Forty-eight healthy subjects were enrolled in each study and received at least 1 dose of treatment. In study 1, 33 subjects (69\%) completed all 3 treatment periods. Fifteen subjects withdrew from the study: 10 (21\%) due to the $\mathrm{AE}$ of vomiting (per protocol), $1(2 \%)$ each due to anemia and streptococcal pharyngitis, and $3(6 \%)$ because of a family emergency. In study 2, 24 subjects (50\%) completed all 4 treatment periods. Twenty-four subjects withdrew from the study: $22(46 \%)$ due to vomiting (per protocol), 1 (2\%) because withdrawal criteria were met (positive urinary drug screen at check-in), and 1 (2\%) of these subjects did not respond to attempts to follow-up on the resolution of AEs. Table 1 presents demographics and baseline characteristics of all dosed subjects in both studies.

Oxycodone Pharmacokinetics: Table 2 presents estimates of the oxycodone PK parameters on day 1 and day 5 at steady state.

Day 1: In both studies, no lag $\left(\mathrm{t}_{\mathrm{lag}=} 0\right)$ was observed between the first dose administration of any treatment on day 1 and the detection of oxycodone in plasma. Day 1 mean oxycodone $\mathrm{AUC}_{0-12 \mathrm{~h}}$ and $\mathrm{C}_{\max }$ increased proportionally with 1 - and 2-tablet doses of OC/APAP ER. In study 1 , mean oxycodone $\mathrm{AUC}_{0-12 \mathrm{~h}}$ was similar for the 2-tablet dose of OC/APAP ER and an equivalent dose (administered as 7.5 $\mathrm{mg} / 325 \mathrm{mg}$ q6h for a total of $15 \mathrm{mg} / 650 \mathrm{mg}$ over a 12-hour period) of IR OC/APAP, whereas $\mathrm{C}_{\max }$ was higher for IR OC/APAP. In study 2, oxycodone $\mathrm{AUC}_{0-12 \mathrm{~h}}$ and $\mathrm{C}_{\max }$ were similar for equivalent doses of $\mathrm{OC} /$ APAP ER (15 mg/650 mg) administered every 12 hours and IR OC/

\begin{tabular}{|l|l|l|}
\hline & Study 1 & Study 2 \\
\hline Characteristic & $\begin{array}{l}\text { All Dosed Subjects } \\
\text { (N=48) }\end{array}$ & $\begin{array}{l}\text { All Dosed Subjects } \\
\text { (N=48) }\end{array}$ \\
\hline Age, $\mathrm{y}$ & & \\
\hline Mean (SD) & $32.1(10.1)$ & $31.1(9.13)$ \\
\hline Range & $18-51$ & $18-51$ \\
\hline Sex, $\mathrm{n}$ (\%) & & $27(56.3)$ \\
\hline Male & $24(50.0)$ & $21(43.8)$ \\
\hline Female & $24(50.0)$ & \\
\hline Race, $\mathrm{n}$ (\%) & & $33(68.8)$ \\
\hline White & $32(66.7)$ & $15(31.3)$ \\
\hline Black or African American & $14(29.2)$ & --- \\
\hline Asian & $1(2.1)$ & --- \\
\hline Multiracial & $1(2.1)$ & \\
\hline Ethnicity, $\mathrm{n}$ (\%) & & $22(45.8)$ \\
\hline Hispanic or Latino & $18(37.5)$ & $26(54.2)$ \\
\hline Not Hispanic or Latino & $30(62.5)$ & $72.9(8.0)$ \\
\hline Weight, kg, mean (SD) & $73.0(9.9)$ & $25.5(2.6)$ \\
\hline Body mass index, kg/m² & & $19.6-29.8$ \\
\hline Mean (SD) & $25.7(2.6)$ & \\
\hline Range & $20.6-29.8$ & \\
\hline \multicolumn{2}{|c|}{ Table 1: Demographics and baseline characteristics. } \\
\hline
\end{tabular}


Citation: Devarakonda K, Morton T, Giuliani M, Kostenbader K, Barrett T (2014) Steady-State Pharmacokinetics of MNK-795, an Extended-Release Oxycodone and Acetaminophen Combination Analgesic: Results from 2 Active Comparator Studies. J Bioequiv Availab 6: 053-060. doi:10.4172/jbb.1000180

\begin{tabular}{|c|c|c|c|c|c|c|}
\hline \multirow[b]{2}{*}{ Parameter, mean (SD) } & \multicolumn{3}{|c|}{ Study $1(n=33)$} & \multicolumn{3}{|c|}{ Study 2 (n=24) } \\
\hline & $\begin{array}{l}\text { OC/APAP ER } \\
1 \text { tablet q12h }\end{array}$ & $\begin{array}{l}\text { OC/APAP ER } \\
2 \text { tablets q12h }\end{array}$ & $\begin{array}{l}\text { IR OC/APAP } \\
1 \text { tablet q6h }\end{array}$ & $\begin{array}{l}\text { OC/APAP ER } \\
2 \text { tablets q12h }\end{array}$ & $\begin{array}{l}\text { IR Oxycodone } \\
1 \text { tablet q6h }\end{array}$ & $\begin{array}{l}\text { IR OC/APAP } \\
1 \text { tablet q6h }\end{array}$ \\
\hline \multicolumn{7}{|l|}{ Day 1} \\
\hline $\mathrm{AUC}_{0-12 h}, \mathrm{ng} \cdot \mathrm{h} / \mathrm{mL}$ & $66.9(15.1)$ & $135.9(30.8)$ & $141.7(29.8)$ & $136.1(23.7)$ & $242.6(19.9)$ & $132.5(22.8)$ \\
\hline $\mathrm{C}_{\max }, \mathrm{ng} / \mathrm{mL}$ & $8.34(2.37)$ & $17.05(3.97)$ & $21.93(4.80)$ & $16.04(3.64)$ & $34.78(8.64)$ & $19.83(5.07)$ \\
\hline$T_{\max }, h^{a}$ & $3.0(0.75-7.0)$ & $3.0(0.5-5.9)$ & $7.0(0.5-8.0)$ & $3.0(0.5-8.0)$ & $7.0(0.75-10.0)$ & $8.0(0.5-10.0)$ \\
\hline$t_{\text {lag }}, h^{a}$ & $0.0(0.0-0.5)$ & $0.0(0.0-0.3)$ & $0.0(0.0-0.25)$ & $0.0(0.0-0.27)$ & $0.0(0.0-0.25)$ & $0.0(0.0-0.25)$ \\
\hline \multicolumn{7}{|l|}{ Day 5 (steady state) } \\
\hline$A \cup C_{0-12 h s s}, n g \cdot h / m L$ & $102.4(29.3)$ & $208.6(59.3)$ & $208.9(57.3)$ & $208.3(45.3)$ & $376.9(83.9)$ & $191.5(42.8)$ \\
\hline $\mathrm{C}_{\text {avgss}}, \mathrm{ng} / \mathrm{mL}$ & $8.53(2.44)$ & $17.38(4.94)$ & $17.41(4.78)$ & $17.36(3.78)$ & $31.41(6.99)$ & $15.96(3.57)$ \\
\hline $\mathrm{C}_{\text {maxss }}, \mathrm{ng} / \mathrm{mL}$ & $12.67(3.48)^{b}$ & $25.67(7.49)^{b}$ & $30.50(8.91)$ & $24.00(5.38)$ & $45.15(10.54)$ & $26.32(6.18)$ \\
\hline $\mathrm{C}_{\text {minss }}, \mathrm{ng} / \mathrm{mL}$ & $4.06(1.40)$ & $8.98(3.52)$ & $8.78(3.17)$ & $9.31(2.39)$ & $19.91(4.93)$ & $8.81(2.40)$ \\
\hline Degree of fluctuation, \% & $101.7(14.1)^{b}$ & $97.2(18.8)^{b}$ & $126.8(27.9)$ & $83.9(17.6)^{b}$ & $79.9(19.8)$ & $110.9(33.4)$ \\
\hline Swing & $2.2(0.6)^{b}$ & $2.0(0.7)^{\mathrm{b}}$ & $2.7(0.9)$ & $1.7(0.6)^{\mathrm{b}}$ & $1.3(0.5)$ & $2.1(0.9)$ \\
\hline$T_{\text {maxss }}, h^{a}$ & $2.0(0.5-10.0)^{\mathrm{b}}$ & $2.0(0.5-7.0)^{b}$ & $6.5(0.5-8.0)$ & $3.0(1.0,5.9)^{\mathrm{b}, \mathrm{c}}$ & $3.0(1.0,12.0)$ & $7.3(0.5,8.1)$ \\
\hline$t_{1 / 2}, h$ & $5.5(1.2)$ & $6.1(1.5)^{\mathrm{b}}$ & $5.5(1.7)$ & $5.4(0.9)^{\mathrm{b}, \mathrm{c}}$ & $4.6(0.6)$ & $4.7(0.6)$ \\
\hline $\mathrm{K}_{\mathrm{el}}, 1 / \mathrm{h}$ & $0.1326(0.0269)$ & $0.1199(0.0291)^{b}$ & $1.1387 \quad(0.0418)$ & $0.1318(0.0223)^{b, c}$ & $0.1525(0.0206)$ & $0.1517(0.0205)$ \\
\hline
\end{tabular}

${ }^{a}$ Median (range); ${ }^{b}$ Reached statistical significance vs IR OC/APAP; ${ }^{R}$ Reached statistical significance vs IR oxycodone

APAP, acetaminophen; $\mathrm{AUC}_{0-12 \mathrm{hss}}$, area under the plasma concentration-time curve from time 0 to $12 \mathrm{~h}$ at steady state; $\mathrm{C}_{\text {avgss }}$, average observed plasma concentration during the dosing interval at steady state; $\mathrm{C}_{\text {maxs }}$, maximum observed plasma concentration at steady state; $\mathrm{C}_{\text {minss }}$, plasma concentration obtained at predose during steady state; $\mathrm{ER}$, extended release; IR, immediate release; $\mathrm{K}_{\mathrm{el}}$, apparent terminal elimination rate constant; OC, oxycodone; $T_{\text {maxss }}$, time to $\mathrm{C}_{\max }$ at steady state; $\mathrm{t}_{1 / 2,}$, terminal elimination half-life

Table 2: Day 1 and steady-state (day 5) pharmacokinetic estimates for oxycodone.

APAP $(7.5 \mathrm{mg} / 325 \mathrm{mg})$ administered every 6 hours and lower for OC/ APAP ER (15 mg/650 mg) administered every 12 hours compared with IR oxycodone $(15 \mathrm{mg})$ administered every 6 hours $(30 \mathrm{mg}$ OC over a 12-hour period; Table 2). In both studies, oxycodone $\mathrm{T}_{\max }$ was 3 hours after the first administration of OC/APAP E

Steady-State (Day 5): In study 1, steady-state oxycodone plasma concentrations were achieved on day $4(\mathrm{P}=0.774)$ and day $3(\mathrm{P}=0.267)$ for the 1- and 2-tablet doses of OC/APAP ER, respectively, whereas steady-state was not attained for IR OC/APAP $(\mathrm{P}<0.05)$. For the 2-tablet dose of OC/APAP ER, $\mathrm{C}_{\min }$ was above $10 \mathrm{ng} / \mathrm{mL}$ on each day in study 1 . In study 2, steady-state oxycodone concentrations were attained within 24 hours after initial administration of OC/APAP ER $(\mathrm{P}=0.138)$ and IR OC/APAP ( $\mathrm{P}=0.144)$. Figure 1A (study 1 ) and $1 \mathrm{~B}$ (study 2 ) present steady-state plasma oxycodone concentrations after the final dose of OC/APAP ER on day 5. At steady-state, mean oxycodone plasma concentrations increased rapidly after administration of OC/APAP ER and were sustained throughout the dosing interval. Oxycodone plasma concentrations were approximately $37 \%$ of peak levels by 12 hours after dosing and remained detectable in plasma for more than 24 hours after the final dose in study 2

In study 1 , the $90 \%$ CIs of the geometric LS means ratios for steadystate (day 5) dose normalized oxycodone PK assessments were within the no difference range for the comparison between the 1- and 2-tablet doses of OC/APAP ER for $\mathrm{AUC}_{0-12 \mathrm{hss}}, \mathrm{C}_{\text {maxss }}, \mathrm{C}_{\text {minss }}, \mathrm{C}_{\text {avgss }}$, and DFL, indicating dose proportionality with respect to oxycodone exposure. In addition, the $90 \%$ CIs for $\mathrm{AUC}_{0-12 \mathrm{hss}}, \mathrm{C}_{\text {minss }}$, and $\mathrm{C}_{\text {avgss }}$ were completely within the no difference range $(80 \%-125 \%)$ for the comparison between both doses OC/APAP ER and IR OC/APAP, indicating similar overall oxycodone bioavailability between these treatments at steady-state. The findings in study 2 also showed similar oxycodone bioavailability for dose-normalized OC/APAP ER, IR OC/APAP, and IR oxycodone at steady-state, as demonstrated by the $90 \%$ CIs of the geometric LS means ratios being fully contained within the range of $80 \%$ to $125 \%$ for oxycodone $\mathrm{AUC}_{0-12 \mathrm{hss}}, \mathrm{C}_{\text {maxss }}, \mathrm{C}_{\text {avgss }}$, and $\mathrm{C}_{\text {minss }}$. In study 1 (Day 5), intrasubject variability $(\mathrm{CV})$ was about $11 \%$ for $\mathrm{AUC}_{0-12 \mathrm{hss}}, 15 \%$ for $\mathrm{C}_{\text {maxss }}$, and $18 \%$ for $\mathrm{C}_{\text {minss }}$, respectively, following administration of 1 or 2 OC/APAP ER tablets. Intersubject variability $(\mathrm{CV})$ for both $\mathrm{AUC}_{0-12}$ hss and $\mathrm{C}_{\text {maxss }}$ was about $28 \%$ and, ranged from $35 \%$ to $39 \%$ for $\mathrm{C}_{\text {minss' }}$, respectively, for oxycodone following administration of 1 or 2 tablets of OC/APAP ER. Following multiple doses of 2 tablets of OC/APAP ER in study 2 (Day 5), intrasubject variability was $12 \%$ for $\mathrm{AUC}_{0-12 \text { hss }}$ and $18 \%$ for both $\mathrm{C}_{\text {myxs }}$, and $\mathrm{C}_{\text {mins, }}$, respectively. Intersubject variability was $22 \%$ for both $\mathrm{AUCC}_{0-12 \text { hss }}$ and $\mathrm{C}_{\text {maxss }}$ and was $26 \%$ for $\mathrm{C}_{\text {minss }}$, respectively.

PK variables that did demonstrate significant differences in study 1 included $\mathrm{C}_{\text {maxs }}$ swing, and DFL, which were $17 \%, 15 \%$, and $19 \%$ lower with the 1-tablet dose and $16 \%, 24 \%$, and $23 \%$ lower with the 2-tablet dose, respectively, compared with IR OC/APAP. The LS means ratios (90\% CI) for $\mathrm{C}_{\text {maxss }}$, swing, and DFL between OC/APAP ER (1 tablet) and IR OC/APAP were $82.9 \%$ (77.8\%-88.2\%), 81.5\% (76.0\%-87.3\%), and $85.3 \%$ (75.7\%-96.1\%), respectively; and between OC/APAP ER (2 tablets) and IR OC/APAP were $83.7 \%$ (78.6\%-89.1\%), 75.6\% (67.1\%$85.2 \%)$, and $76.9 \%(71.7 \%-82.5 \%)$, respectively. $\mathrm{T}_{\text {maxss }}$ was similar between the 1- and 2-tablet doses of OC/APAP ER, but significantly shorter than IR OC/APAP and IR oxycodone administered every 6 hours $(P<0.05)$ as maximum concentrations of these IR products occurred after the second dose on Day 5 (Table 2). For 2-tablets of OC/ APAP ER, the $t_{1 / 2}$ of oxycodone was significantly longer and $K_{e l}$ was significantly slower than for IR OC/APAP $P<0.05)$.

For study 2, DFL of oxycodone concentrations with OC/APAP ER during the 12-hour dosing interval was similar to IR oxycodone administered every 6 hours but $23 \%$ less than that from IR OC/APAP administered every 6 hours (77.3\%: 90\% CI, 69.3\%-86.3\%). Swing in oxycodone concentrations for OC/APAP ER was also statistically smaller than IR OC/APAP (80.0\%: 90\% CI, 67.6\%-94.6\%). LS mean $\mathrm{T}_{\text {maxss }}$ was significantly shorter for OC/APAP ER than IR OC/APAP and IR oxycodone administered every 6 hours $(P<0.05)$, which were observed after the second dose on day 5 . The $t_{1 / 2}$ of oxycodone from OC/APAP ER ( $5.5 \mathrm{~h})$ was approximately 1 hour longer than that from IR oxycodone ( $4.7 \mathrm{~h})$ or IR OC/APAP (4.6 h; $P<.001$ for both). 
Citation: Devarakonda K, Morton T, Giuliani M, Kostenbader K, Barrett T (2014) Steady-State Pharmacokinetics of MNK-795, an Extended-Release Oxycodone and Acetaminophen Combination Analgesic: Results from 2 Active Comparator Studies. J Bioequiv Availab 6: 053-060. doi:10.4172/jbb.1000180

Acetaminophen Pharmacokinetics: Table 3 presents estimates of the acetaminophen PK parameters on day 1 and day 5 at steady state.

Day 1: In both studies there was also no lag in the appearance of acetaminophen in plasma following the administration of any treatment on day 1 . Acetaminophen $\mathrm{T}_{\max }$ was reached within 1 hour for each treatment in both studies with the exception of IR tramadol/ APAP in study 2 , in which acetaminophen $\mathrm{T}_{\max }$ was reached at 0.75 hours after the second dose. In study 1 , mean acetaminophen $\mathrm{AUC}_{0-12 \mathrm{~h}}$ and $\mathrm{C}_{\text {max }}$ were proportional with the 1- and 2-tablet doses of OC/APAP ER. In addition, acetaminophen $\mathrm{AUC}_{0-12 \mathrm{~h}}$ and $\mathrm{C}_{\max }$ for IR OC/APAP were similar to that of the 2-tablet dose of OC/APAP ER. In study 2, mean day 1 acetaminophen $\mathrm{AUC}_{0-12 \mathrm{~h}}$ and $\mathrm{C}_{\max }$ were comparable between all 3 treatments (Table 3 ).

Steady-State (Day 5): In study 1, steady-state acetaminophen concentrations were achieved on day 4 for the 1-tablet dose $(\mathrm{P}=0.787)$ of OC/APAP ER and on day 2 for the 2-tablet dose $(\mathrm{P}=0.188)$ of $\mathrm{OC} /$ APAP ER and IR OC/APAP ( $\mathrm{P}=0.675)$. Steady-state acetaminophen concentrations in study 2 were reached within 24 hours after initial administration of OC/APAP ER $(\mathrm{P}=0.089)$ and IR tramadol/APAP $(\mathrm{P}=0.959)$, and within 12 hours for IR OC/APAP $(\mathrm{P}=0.052)$. In

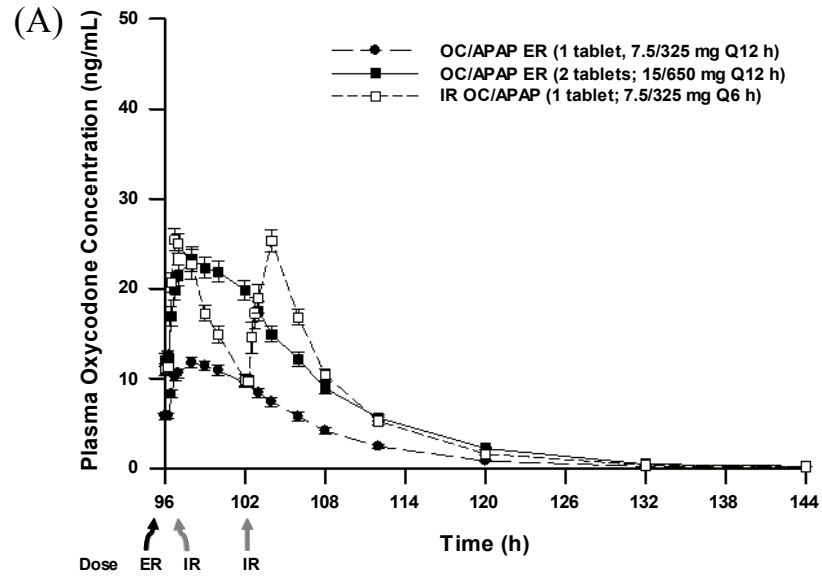

(B)

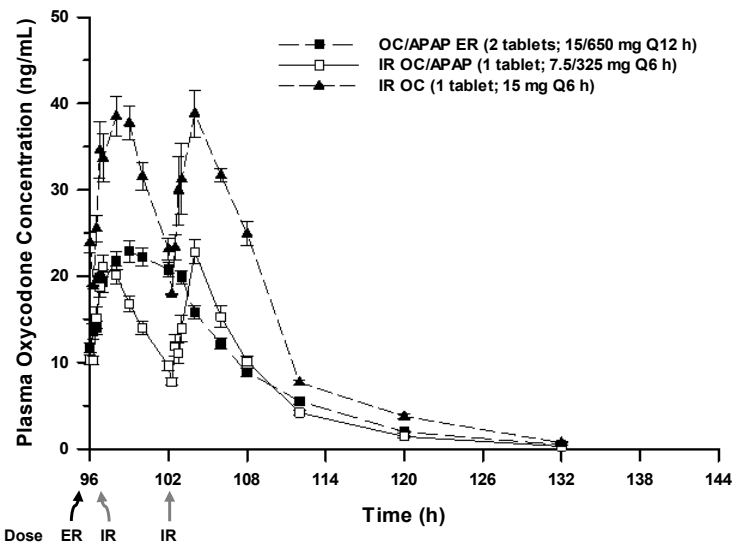

Figure 1: A. Mean (SE) steady-state plasma concentration of oxycodone after the last dose of OC/APAP ER in study 1. B. Mean (SE) steady-state plasma concentration of oxycodone after the last dose of OC/APAP ER in study 2 (B). Arrows indicate timing of doses.
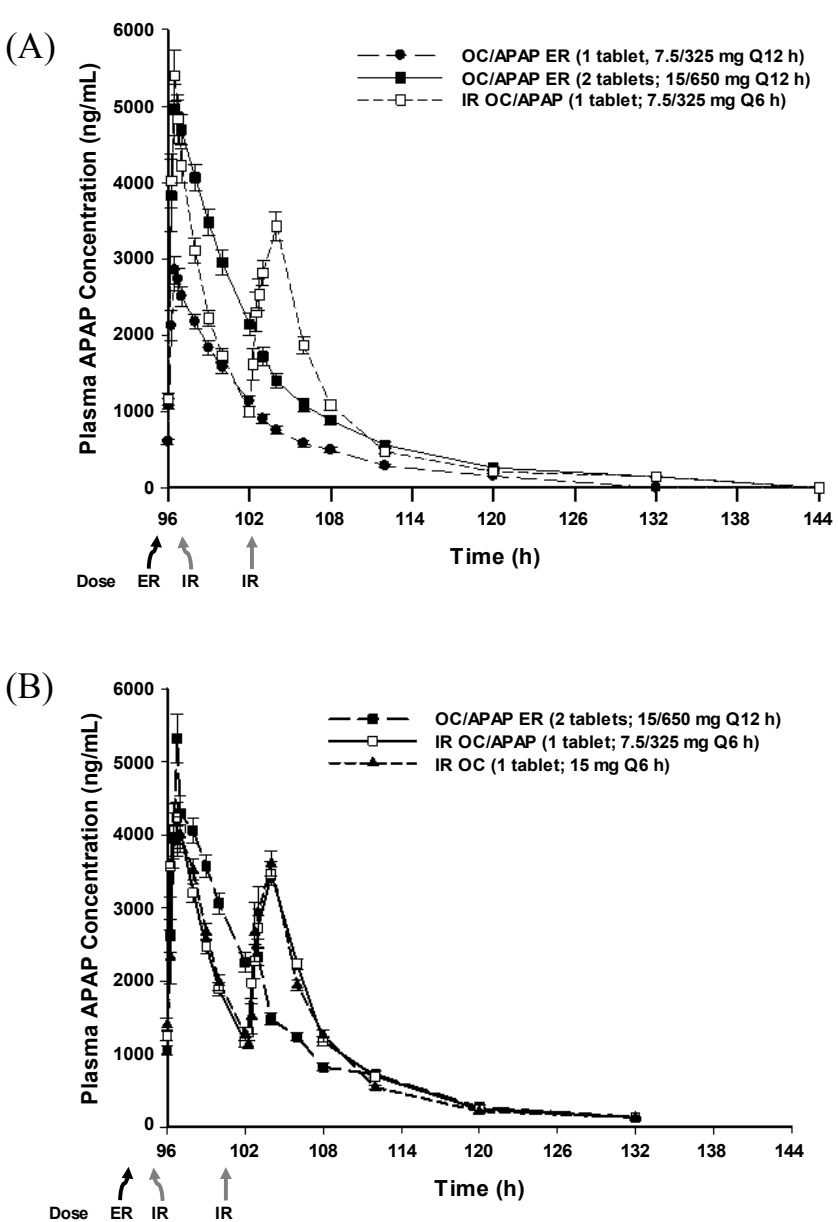

Figure 2: A. Mean (SE) steady-state plasma concentration of acetaminophen after the last dose of OC/APAP ER in study 1. B. Mean (SE) steady-state plasma concentration of acetaminophen after the last dose of OC/APAP ER in study 2 (B).

addition, $\mathrm{C}_{\min }$ values for both doses of OC/APAP ER were below those for each comparator in both studies. Figure 2A (study 1) and 2B (study 2) present steady-state acetaminophen plasma concentrations after the final dose of OC/APAP ER on day 5

In both studies, acetaminophen $\mathrm{T}_{\text {maxs }}$ was reached within 1 hour after administration of each study medication (Table 3). At steady-state (day 5), mean acetaminophen plasma concentrations from OC/APAP ER increased rapidly after administration and declined to levels below those of the comparators by 7 to 12 hours after the initial dose on day 5. In study 1 , the comparisons of geometric LS means ratios for the results for dose-normalized acetaminophen $A_{U C} C_{0-12 h s,} C_{\text {maxss }}, C_{\text {minss, }}$ $\mathrm{C}_{\text {avgss }}$, DFL, and swing found no differences between OC/APAP ER and the comparators from each study, as indicated by the $90 \%$ CIs being within the no difference range. However, in study 2, acetaminophen $\mathrm{C}_{\text {minss }}$ after administration of OC/APAP ER was $21 \%$ lower than that observed after IR tramadol/APAP and 22\% lower than after IR OC/ APAP (LS means ratios [90\% CI] of 79.1\% [73.4\%-85.4\%] and $78.4 \%$ [72.8\%-84.5\%], respectively). In addition, swing in acetaminophen plasma concentrations was higher for higher for OC/APAP ER than for tramadol/APAP and IR OC/APAP, with LS means ratios (90\% CI) of $127.6 \%$ (106.1\%-153.5\%) and $132.6 \%$ (110.5\%-159.3\%). In study 1 , acetaminophen $t_{1 / 2}$ was longer for the 2-tablet dose of OC/APAP ER 
Citation: Devarakonda K, Morton T, Giuliani M, Kostenbader K, Barrett T (2014) Steady-State Pharmacokinetics of MNK-795, an Extended-Release Oxycodone and Acetaminophen Combination Analgesic: Results from 2 Active Comparator Studies. J Bioequiv Availab 6: 053-060. doi:10.4172/jbb.1000180

compared with IR OC/APAP $(P<0.05)$. In study 2 , acetaminophen $\mathrm{T}_{\max }$ were not significantly different between treatments $(P>0.05)$, and acetaminophen $t_{1 / 2}$ for OC/APAP ER were similar to those for IR OC/ APAP; however, the least squares mean of $\mathrm{t}_{1 / 2}$ was approximately 2.3 hours longer for OC/APAP ER than for IR tramadol/APAP $(P<0.05)$.

Across studies, intrasubject variability $(\mathrm{CV})$ for acetaminophen was $9 \%$ for $\mathrm{AUC}_{0-12 \mathrm{hss}}$, ranged from $19 \%$ to $23 \%$ for $\mathrm{C}_{\text {maxss }}$ and was $15 \%$ for $\mathrm{C}_{\text {minss }}$, respectively, following administration of 1 or 2 OC/APAP ER tablets. Intersubject variability $(\mathrm{CV})$ for acetaminophen ranged from $21 \%$ to $27 \%$ for $\mathrm{AUC}_{0-12 \mathrm{hss}}, 24 \%$ to $33 \%$ for $\mathrm{C}_{\text {maxss }}$ and $32 \%$ to $39 \%$ for $\mathrm{C}_{\text {minss }}$, respectively, after administration of 1 or 2 tablets of OC/APAP ER.

Overall, these findings suggest that acetaminophen exposure was proportional to the administered doses of OC/APAP ER, while producing similar steady-state acetaminophen bioavailability for OC/ APAP ER versus IR OC/APAP and IR tramadol/APAP.

\section{Safety/Tolerability}

Table 4 presents AEs for each treatment condition in both studies. Overall in study 1,42 of 48 subjects (87.5\%) experienced $\geq 1 \mathrm{AE}$ during any condition; 19 participants (47.5\%) experienced an AE while receiving 1 tablet of OC/APAP ER, 29 participants (70.7\%) with 2 tablets of OC/APAP ER, and 30 (73.2\%) after receiving IR OC/APAP (Table 4). No serious AEs (SAEs) were reported during the study. The most common AEs overall were nausea (45.8\%), pruritus (37.5\%), headache $(33.3 \%)$, dizziness $(31.3 \%)$, infrequent bowel movements (20.8\%), vomiting (20.8\%), and somnolence (16.7\%). In general, OC/ APAP ER administered as 1 tablet had fewer AEs than either 2 tablets of OC/APAP ER or IR OC/APAP. No significant clinical differences in safety or tolerability were reported between OC/APAP ER (2 tablets) and IR OC/APAP. Somnolence was numerically greater for both doses of OC/APAP ER compared with IR OC/APAP. Ten participants (21\%) discontinued because of vomiting per protocol specification; 1 during

\begin{tabular}{|c|c|c|c|c|c|c|}
\hline \multirow[b]{2}{*}{ Parameter, mean (SD) } & \multicolumn{3}{|c|}{ Study $1(n=33)$} & \multicolumn{3}{|c|}{ Study $2(n=24)$} \\
\hline & $\begin{array}{l}\text { OC/APAP ER } \\
1 \text { tablet q12h }\end{array}$ & $\begin{array}{l}\text { OC/APAP ER } \\
2 \text { tablets q12h }\end{array}$ & $\begin{array}{l}\text { IR OC/APAP } \\
1 \text { tablet q6h }\end{array}$ & $\begin{array}{l}\text { OC/APAP ER } \\
2 \text { tablets q12h }\end{array}$ & $\begin{array}{l}\text { IR tramadol/APAP } \\
1 \text { tablet q6h }\end{array}$ & $\begin{array}{l}\text { IR OC/APAP } \\
1 \text { tablet q6h }\end{array}$ \\
\hline \multicolumn{7}{|l|}{ Day 1} \\
\hline $\mathrm{AUC}_{0-12 \mathrm{~h}}, \mathrm{ng} \cdot \mathrm{h} / \mathrm{mL}$ & $12192(3331)$ & $24141(6436)$ & $24884(6656)$ & 24924 (5667) & $26343(4721)$ & $25094(5085)$ \\
\hline $\mathrm{C}_{\max }, \mathrm{ng} / \mathrm{mL}$ & $2631(815)$ & $5245(1473)$ & $5146(1553)$ & $4858(1066)$ & $4568(976)$ & $4318(1006)$ \\
\hline$T_{\max }, h^{a}$ & $0.55(0.25-3.0)$ & $0.75(0.25-2.0)$ & $0.50(0.25-8.0)$ & $1.0(0.5-4.0)$ & $6.75(0.5-8.2)$ & $0.53(0.5-8.0)$ \\
\hline$t_{\text {lag }}, h^{a}$ & $0.0(0.0-0.25)$ & $0.0(0.0-0.25)$ & $0.0(0.0-0.0)$ & $0.0(0.0-0.0)$ & $0.0(0.0-0.3)$ & $0.0(0.0-0.0)$ \\
\hline \multicolumn{7}{|l|}{ Day 5 (steady state) } \\
\hline $\mathrm{AUC}_{0-12 \mathrm{hss}}, \mathrm{ng} \cdot \mathrm{h} / \mathrm{mL}$ & $15307(4092)$ & $28512(7714)$ & 28719 (7023) & $28160.40(5807.09)$ & $29711.92(5427.37)$ & $29284.22(5477.73)$ \\
\hline $\mathrm{C}_{\text {avgss }}, \mathrm{ng} / \mathrm{mL}$ & $1276(341)$ & $2376(643)$ & $2393(585)$ & $2346.70(483.92)$ & $2475.99(452.28)$ & $2440.35(456.48)$ \\
\hline $\mathrm{C}_{\operatorname{maxss}}, \mathrm{ng} / \mathrm{mL}$ & $3117(840)$ & $5872(1932)$ & $5968(1639)$ & $4792.50(1132.40)$ & $5078.33(1189.70)$ & $4876.67(1383.08)$ \\
\hline $\mathrm{C}_{\text {minss }}, \mathrm{ng} / \mathrm{mL}$ & $474.67(163)$ & $870.42(336)$ & $922.58(321)$ & $852.75(273.25)^{b, c}$ & $1070.92(367.35)$ & $1069.13(291.83)$ \\
\hline Degree of fluctuation, \% & $212.1(52.3)$ & $218.1(81.1)$ & $213.8(50.5)$ & $169.1(39.8)$ & $163.9(47.2)$ & $155.3(38.8)$ \\
\hline Swing & $6.0(2.0)$ & $6.6(3.6)$ & $5.9(2.2)$ & $5.1(2.1)^{b, c}$ & $4.2(2.1)$ & $3.8(1.6)$ \\
\hline$T_{\text {maxss }}, h^{a}$ & $0.5(0.3-3.0)$ & $0.5(0.3-3.0)$ & $0.5(0.3-8.0)$ & $1.0(0.5,4.0)$ & $0.9(0.3,8.0)$ & $0.8(0.3,8.0)$ \\
\hline$t_{1 / 2}, h$ & $5.6(1.4)$ & $7.5(2.9)^{b}$ & $5.7(3.0)$ & $6.9(1.8)^{c}$ & $5.3(1.1)$ & $6.2(1.8)$ \\
\hline $\mathrm{K}_{\mathrm{el}}, 1 / \mathrm{h}$ & $0.1308(0.0317)$ & $0.1026(0.0292)^{b}$ & $0.1416(0.0515)$ & $0.1072(0.0285)^{\mathrm{c}}$ & $0.1355(0.0279)$ & $0.1201(0.0338)$ \\
\hline
\end{tabular}

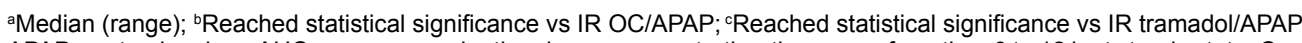

APAP, acetaminophen; $A \cup C_{0-12 h s s}$, area under the plasma concentration-time curve from time 0 to $12 \mathrm{~h}$ at steady state; $\mathrm{C}_{\text {avgss }}$, average observed plasma concentration during the dosing interval at steady state; $\mathrm{C}_{\text {maxs }}$, maximum observed plasma concentration at steady state; $\mathrm{C}_{\text {mos. }}$, plasma concentration obtained at predose during steady state; ER, extended release; IR, immediate release; $K_{e l}$, apparent terminal elimination rate constant; OC, oxycodone; $T_{\operatorname{maxss}}$, time to $C_{\max }$ at steady state; $t_{1 / 2}$ terminal elimination half-life

Table 3: Day 1 and steady-state (day 5) pharmacokinetic estimates for acetaminophen

\begin{tabular}{|c|c|c|c|c|c|c|c|}
\hline \multirow[b]{2}{*}{$A E, n(\%)$} & \multicolumn{3}{|c|}{ Study 1} & \multicolumn{4}{|c|}{ Study 2} \\
\hline & $\begin{array}{c}\text { OCIAPAP ER } \\
1 \text { tablet } q 12 h \\
(n=40)\end{array}$ & $\begin{array}{c}\text { OC/APAP ER } \\
2 \text { tablets } q 12 h(n=41)\end{array}$ & $\begin{array}{c}\text { IR OC/APAP } \\
1 \text { tablet q6h } \\
(n=41)\end{array}$ & $\begin{array}{l}\text { OC/APAP ER } \\
2 \text { tablets q12h } \\
\quad(n=33)\end{array}$ & $\begin{array}{l}\text { IR Oxycodone } \\
1 \text { tablet q6h } \\
(n=34)\end{array}$ & $\begin{array}{l}\text { IR tramadol/APAP } \\
1 \text { tablet } q 6 h \\
(n=28)\end{array}$ & $\begin{array}{c}\text { IR OC/APAP } \\
1 \text { tablet q6h } \\
(n=31)\end{array}$ \\
\hline Subjects with at least $1 \mathrm{TEAE}$ & $19(47.5)$ & $29(70.7)$ & $30(73.2)$ & $15(45.5)$ & $28(82.4)$ & $12(42.9)$ & $20(64.5)$ \\
\hline Nausea & $5(12.5)$ & $12(29.3)$ & $13(31.7)$ & $8(24.2)$ & $13(38.2)$ & $2(7.1)$ & $9(29.0)$ \\
\hline Vomiting & $1(2.5)$ & $5(12.2)$ & $4(9.8)$ & $7(21.2)$ & $8(23.5)$ & $2(7.1)$ & $5(16.1)$ \\
\hline Dizziness & $5(12.5)$ & $6(14.6)$ & $7(17.1)$ & $4(12.1)$ & $13(38.2)$ & $2(7.1)$ & $4(12.9)$ \\
\hline Pruritus & $5(12.5)$ & $10(24.4)$ & $10(24.4)$ & $7(21.2)$ & $13(38.2)$ & $5(17.9)$ & $2(6.5)$ \\
\hline Headache & $9(22.5)$ & $4(9.8)$ & $7(17.1)$ & $5(15.2)$ & $5(14.7)$ & $3(10.7)$ & $3(9.7)$ \\
\hline Feeling hot & 0 & $1(2.4)$ & $3(7.3)$ & $2(6.1)$ & $4(11.8)$ & $1(3.6)$ & $2(6.5)$ \\
\hline Infrequent bowel movements & $4(10.0)$ & $5(12.2)$ & $3(7.3)$ & $0(0.0)$ & $6(17.6)$ & $1(3.6)$ & $2(6.5)$ \\
\hline Abdominal pain & $1(2.5)$ & $3(7.3)$ & $1(2.4)$ & $2(6.1)$ & $0(0.0)$ & $1(3.6)$ & $3(9.7)$ \\
\hline Somnolence & $5(12.5)$ & $5(12.2)$ & $1(2.4)$ & $1(3.0)$ & $1(2.9)$ & $2(7.1)$ & $1(3.2)$ \\
\hline
\end{tabular}

${ }^{a} A E s$ were coded using the Medical Dictionary for Regulatory Activities (MedDRA ${ }^{\circledR}$; MedDRA MSSO, McLean, Virginia, USA)

APAP, acetaminophen; ER, extended release; IR, immediate release; OC, oxycodone; q6h, every 6 hours; q12h, every 12 hours; TEAE, treatment-emergent adverse event

Table 4: Summary of treatment-emergent adverse events ${ }^{a}$. 
Citation: Devarakonda K, Morton T, Giuliani M, Kostenbader K, Barrett T (2014) Steady-State Pharmacokinetics of MNK-795, an Extended-Release Oxycodone and Acetaminophen Combination Analgesic: Results from 2 Active Comparator Studies. J Bioequiv Availab 6: 053-060. doi:10.4172/jbb.1000180

1-tablet OC/APAP ER (7.5/325 mg q12h), 5 during 2-tablet OC/APAP ER (15/650 mg q12h), and 4 during 1-tablet IR OC/APAP (7.5/325 mg q6h).

In study 2, 44 of 48 subjects (91.7\%) experienced $\geq 1 \mathrm{AE}$. More subjects reported AEs during IR oxycodone administration (82.4\%) than during administration of IR OC/APAP (64.5\%), OC/APAP ER (45.5\%), or IR tramadol/APAP (42.9\%) (Table 4). The most common AEs overall were nausea (54.2\%), vomiting (45.8\%), pruritus $(39.6 \%)$, dizziness $(39.6 \%)$, and headache $(29.2 \%)$. As would be expected, AEs that are common to opioids (eg, nausea, vomiting, and dizziness) were low with IR tramadol/APAP and highest with the highest dose of oxycodone (ie, IR oxycodone $15 \mathrm{mg}$ q6h). AEs were similar between OC/APAP ER (15/650 mg q12h) and IR OC/APAP (7.5/325 mg q6h). No SAEs were reported during the study. Twenty-two subjects $(45.8 \%)$ were withdrawn from the study because of vomiting per protocol requirement, 7 (21.2\%) of which occurred during OC/APAP ER treatment.

No apparent clinically significant treatment-related trends in vital sign and pulse oximetry measurements, ECG or physical examination findings were observed in either study. In study 1 , one subject experienced an AE of anemia (during IR OC/APAP), which was accompanied by clinically significant decreases in erythrocytes, hemoglobin, and hematocrit on laboratory assessments. This AE was considered by the investigator to be mild and not related to study medication, and resolved after study discontinuation. In study 2, two participants had laboratory abnormalities that were considered by the investigator to be clinically significant. One subject experienced increased hepatic enzymes (alanine aminotransferase, $227 \mathrm{IU} / \mathrm{L}$; aspartate aminotransferase, $142 \mathrm{IU} / \mathrm{L}$; and gamma-glutamyl transferase, $90 \mathrm{IU} / \mathrm{L}$ ) on day 7 with IR oxycodone, which returned to within the normal range at admission on the next treatment period. These elevations were considered by the investigator to be mild and possibly related to study medication. A second subject had a clinically significant urinalysis result of trichomoniasis during IR tramadol/ APAP, which was considered by the investigator to be mild and not related to study medication. This participant was referred to his or her primary care physician for follow-up. No other clinically significant changes in laboratory measures were noted in either study.

\section{Discussion}

These multiple-dose PK studies show that administering OC/APAP ER every 12 hours resulted in plasma concentrations of oxycodone and acetaminophen comparable to that of the IR formulations administered every 6 hours. In study 1 , steady-state levels of oxycodone and acetaminophen were attained within 2 to 4 days of dosing of OC/ APAP ER, 1- and 2-tablet doses of OC/APAP ER demonstrated dose proportionality, and the relative bioavailabilities of oxycodone and acetaminophen following administration of 1 or 2 tablets of $\mathrm{OC} /$ APAP ER every 12 hours were comparable with that IR OC/APAP (dose normalized) administered every 6 hours. In addition, this study demonstrated lower peak oxycodone concentrations and less fluctuation in oxycodone concentrations with OC/APAP ER (administered q12h) compared with IR OC/APAP (administered q6h). In study 2, steadystate plasma concentrations of oxycodone and acetaminophen were achieved within 24 hours after initial administration of OC/APAP ER. Under steady-state conditions, overall bioavailability of oxycodone and acetaminophen with OC/APAP ER administered $\mathrm{q} 12 \mathrm{~h}$ was similar to that of the IR products administered q6h; however, less fluctuation in oxycodone plasma concentrations occurred with OC/APAP ER compared with IR OC/APAP.

Both studies also showed that after dosing OC/APAP ER, plasma levels of oxycodone and acetaminophen rose quickly with no lag in appearance, and oxycodone concentrations decreased more slowly than acetaminophen concentrations. After the last dose during steady state, acetaminophen plasma levels tapered off to levels below IR comparators approximately 7 to 12 hours after the last dose of OC/APAP ER. In two separate single-dose studies that fully characterized plasma concentration-time data for oxycodone and APAP, administration of OC/APAP ER showed similar results (K. Devarakonda, PhD, et al., J. Bioequivalence \& Bioavailability, 2014 (In Press)). In addition to the tapering of acetaminophen concentrations after the final dose, in study 2, steady-state trough acetaminophen plasma concentrations after OC/APAP ER were $21 \%$ lower than after IR tramadol/APAP and 22\% lower than after IR OC/APAP, suggesting a low level of acetaminophen prior to the next dose of OC/APAP ER. This effect is a function of the acetaminophen release characteristics of OC/APAP ER. In addition, OC/APAP ER tablets contain $325 \mathrm{mg}$ of acetaminophen, and when administered as 2 tablets every 12 hours, the total amount of acetaminophen $(1300 \mathrm{mg})$ remains below the daily dose limit recommended by the US Food and Drug Administration [30].

In these 2 cross-over studies, following administration of 1 or 2 tablets of OC/APAP ER the intrasubject variability (within subject) in $\mathrm{AUC}_{0-12 \mathrm{hss}}, \mathrm{C}_{\text {maxss }}$, and $\mathrm{C}_{\text {minss }}$ remained low for both oxycodone and APAP. Similarly, intersubject variability (across subjects) for AUC0-12 hss and $\mathrm{C}_{\text {maxs }}$ remained low. However, the overall minimum plasma concentration $\left(\mathrm{C}_{\text {minss }}\right)$ for oxycodone and APAP in both studies was relatively higher (range $32 \%$ to $39 \%$ ) with the exception of $C_{\text {minss }}$ for Study 2 that was $20 \%$ to $39 \%$ lower than in Study 1 . This difference may be attributed to the variability in actual blood draw times prior to the $96 \mathrm{~h}$ timepoint in Study 1.

In these PK studies, OC/APAP ER provided consistent plasma levels with twice-daily (every 12 hours) administration. Multiple OC/APAP ER doses were generally well tolerated in healthy adult participants and, although the study population was small, no SAEs occurred. AEs occurring after administration of OC/APAP ER were similar to those occurring after administration of IR OC/APAP and IR tramadol/APAP and less frequent than after administration of IR OC. In both studies, the most common AEs affected the gastrointestinal and central nervous systems, consistent with those reported with opioid analgesics [6,31].

As typical with PK studies, the generalizability of these results is limited by the population enrolled (healthy volunteers) and the small sample size. In addition, using agents in a controlled clinical trial setting may not be representative of real-world dosing, particularly due to high medication adherence, rigidly timed administration, and continuous AE monitoring.

\section{Conclusions}

In conclusion, these multiple-dose $\mathrm{PK}$ studies demonstrated that at steady-state, 12-hour dosing of OC/APAP ER yielded plasma concentrations comparable to those of IR products administered every 6 hours, but with less fluctuation in plasma levels of oxycodone and lower trough plasma concentrations of acetaminophen. In addition, OC/APAP ER demonstrated dose proportionality between 1-tablet 
Citation: Devarakonda K, Morton T, Giuliani M, Kostenbader K, Barrett T (2014) Steady-State Pharmacokinetics of MNK-795, an Extended-Release Oxycodone and Acetaminophen Combination Analgesic: Results from 2 Active Comparator Studies. J Bioequiv Availab 6: 053-060. doi:10.4172/jbb.1000180

and 2-tablet administrations. The adverse events occurring in this study were as would be expected for this class of medication and type of study.

\section{Acknowledgments}

Technical editorial and medical writing support for the development of this manuscript was provided by Lisa Bergstrom, PhD, James Bergstrom, $\mathrm{PhD}$, and Mary Tom, PharmD, Synchrony Medical Communications, LLC, West Chester, PA Funding for the technical editorial and medical writing support was provided by Mallinckrodt Inc., Hazelwood, MO.

\section{References}

1. Dubois MY, Gallagher RM, Lippe PM (2009) Pain medicine position paper. Pain Med 10: 972-1000.

2. Number of all-listed procedures for discharges from short-stay hospitals, by procedure category and age: United States, 2010. May 30, 2013.

3. National Center For Health Statistics Ambulatory and Hospital Care Statistics Branch. National Ambulatory Medical Care Survey: 2010 Summary Tables. 2013.

4. Pletcher MJ, Kertesz SG, Kohn MA, Gonzales R (2008) Trends in opioid prescribing by race/ethnicity for patients seeking care in US emergency departments. JAMA 299: 70-78.

5. Apfelbaum JL, Chen C, Mehta SS, Gan TJ (2003) Postoperative pain experience: results from a national survey suggest postoperative pain continues to be undermanaged. Anesth Analg 97: 534-540.

6. Sinatra $R(2010)$ Causes and consequences of inadequate management of acute pain. Pain Med 11: 1859-1871.

7. Daniels S, Casson E, Stegmann JU, Oh C, Okamoto A, et al. (2009) A randomized, double-blind, placebo-controlled phase 3 study of the relative efficacy and tolerability of tapentadol IR and oxycodone IR for acute pain. Curr Med Res Opin 25: 1551-1561.

8. McCarberg BH (2011) Pain management in primary care: strategies to mitigate opioid misuse, abuse, and diversion. Postgrad Med 123: 119-130.

9. Ordoñez Gallego A, González Barón M, Espinosa Arranz E (2007) Oxycodone: a pharmacological and clinical review. Clin Transl Oncol 9: 298-307.

10. Lugo RA, Kern SE (2004) The pharmacokinetics of oxycodone. J Pain Palliat Care Pharmacother 18: 17-30.

11. McCarberg BH, Barkin RL (2001) Long-acting opioids for chronic pain pharmacotherapeutic opportunities to enhance compliance, quality of life, and analgesia. Am J Ther 8: 181-186.

12. Amabile CM, Bowman BJ (2006) Overview of oral modified-release opioid products for the management of chronic pain. Ann Pharmacother 40: 13271335.

13. Smith HS (2009) Potential analgesic mechanisms of acetaminophen. Pain Physician 12: 269-280.

14. Gammaitoni AR, Galer BS, Lacouture P, Domingos J, Schlagheck T (2003) Effectiveness and safety of new oxycodone/acetaminophen formulations with reduced acetaminophen for the treatment of low back pain. Pain Med 4: 21-30.

15. Gatti A, Sabato E, Di Paolo AR, Mammucari M, Sabato AF (2010) Oxycodone/ paracetamol: a low-dose synergic combination useful in different types of pain. Clin Drug Investig 30: 3-14.

16. Raffa RB, Pergolizzi JV, Segarnick DJ, Tallarida RJ (2010) Oxycodone combinations for pain relief. Drugs Today (Barc) 46: 379-398.

17. Raffa RB (2001) Pharmacology of oral combination analgesics: rational therapy for pain. J Clin Pharm Ther 26: 257-264.
18. Raffa RB, Pergolizzi JV, Jr. (2012) Multi-mechanistic analgesia for opioidinduced hyperalgesia. J Clin Pharm Ther 37: 125-127.

19. Cooper SA, Precheur H, Rauch D, Rosenheck A, Ladov M, et al. (1980) Evaluation of oxycodone and acetaminophen in treatment of postoperative dental pain. Oral Surg Oral Med Oral Pathol 50: 496-501.

20. Palangio M, Morris E, Doyle RT, Jr., Dornseif BE, Valente TJ (2002) Combination hydrocodone and ibuprofen versus combination oxycodone and acetaminophen in the treatment of moderate or severe acute low back pain. Clin Ther 24: 87-99.

21. Caldwell JR, Hale ME, Boyd RE, Hague JM, Iwan T, et al. (1999) Treatment of osteoarthritis pain with controlled release oxycodone or fixed combination oxycodone plus acetaminophen added to nonsteroidal antiinflammatory drugs: a double blind, randomized, multicenter, placebo controlled trial. J Rheumatol 26: 862-869.

22. Corsinovi L, Martinelli E, Fonte G, Astengo M, Sona A, et al. (2009) Efficacy of oxycodone/acetaminophen and codeine/acetaminophen vs. conventional therapy in elderly women with persistent, moderate to severe osteoarthritisrelated pain. Arch Gerontol Geriatr 49: 378-382.

23. Gatti A, Sabato AF, Carucci A, Bertini L, Mammucari M, et al. (2009) Adequacy assessment of oxycodone/paracetamol (acetaminophen) in multimodal chronic pain : a prospective observational study. Clin Drug Investig 29: 31-40.

24. Raffaeli W, Pari C, Corvetta A, Sarti D, Di S, V, et al. (2010) Oxycodone/ acetaminophen at low dosage: an alternative pain treatment for patients with rheumatoid arthritis. J Opioid Manag 6: 40-46.

25. Sima L, Fang WX, Wu XM, Li F (2012) Efficacy of oxycodone/paracetamo for patients with bone-cancer pain: a multicenter, randomized, double-blinded, placebo-controlled trial. J Clin Pharm Ther 37: 27-31.

26. Gaskell H, Derry S, Moore RA, McQuay HJ (2009) Single dose oral oxycodone and oxycodone plus paracetamol (acetaminophen) for acute postoperative pain in adults. Cochrane Database Syst Rev: CD002763.

27. Korn S, Vassil TC, Kotey PN, Fricke JR, Jr. (2004) Comparison of rofecoxib and oxycodone plus acetaminophen in the treatment of acute pain: a randomized double-blind, placebo-controlled study in patients with moderate to severe postoperative pain in the third molar extraction model. Clin Ther 26: 769-778.

28. Palangio M, Wideman GL, Keffer M, Landau CJ, Morris E, et al. (2000) Combination hydrocodone and ibuprofen versus combination oxycodone and acetaminophen in the treatment of postoperative obstetric or gynecologic pain Clin Ther 22: 600-612.

29. Muir KT, Gomeni RO (2004) Non-compartmental analysis. In: Bonate, P.L., Howard, D.R., eds. Pharmacokinetics in Drug Development: Clinical Study Design and Analysis. 1 ed. AAPS Press, Arlington, VA, pp 235-265.

30. Kux L (2011) Prescription drug products containing acetaminophen; action to reduce liver injury from unintentional overdose. Federal Register 76: 2691 2697

31. Moskovitz BL, Benson CJ, Patel AA, Chow W, Mody SH, et al. (2011) Analgesic treatment for moderate-to-severe acute pain in the United States: patients perspectives in the Physicians Partnering Against Pain $\left(\mathrm{P}^{3}\right)$ survey. J Opioid Manag 7: 277-286. 